\title{
Comparison of Renal Responses to Cyclophosphamide and Mycophenolate Mofetil used as Induction Therapies in Korean Patients with Lupus Nephritis
}

\author{
Sung-Eun Choi ${ }^{1, *}$, Dong-Jin Park ${ }^{1, *}$, Ji-Hyoun Kang ${ }^{1}$, Kyung-Eun Lee $^{1}$, Haimuzi Xu ${ }^{1}$, Ji Shin Lee ${ }^{2}$, Yoo-Duk Choi ${ }^{2}$, \\ Shin-Seok Lee ${ }^{1}$ \\ ${ }^{1}$ Division of Rheumatology, Department of Internal Medicine, and ${ }^{2}$ Department of Pathology, Chonnam National University Medical School \& \\ Hospital, Gwangju, Korea
}

\begin{abstract}
Objective. Although intravenous cyclophosphamide (IVC) is generally accepted as the standard therapy for induction treatment of active proliferative lupus nephritis (LN), several clinical trials have suggested that mycophenolate mofetil (MMF) is at least as effective as IVC. Because few Asian studies have compared the two treatment modalities, we compared the efficacies of MMF and IVC as LN remission induction treatments in Korean patients. Methods. We enrolled 39 patients with class III and IV LN who received MMF or IVC as LN induction therapy. The renal outcomes (i.e., complete response [CR], partial response [PR], and no response [NR]) at 6 and 12 months were defined using the ACR 2006 response criteria. Results. Of 39 patients, 23 (59.0\%) were treated with IVC, and 16 (41.0\%) were treated with MMF. Demographics, clinical characteristics, laboratory data, and adverse events did not significantly differ between the two groups. However, C3 levels were lower and activity scores in renal biopsy were higher in IVC-treated patients. CRs were achieved by $11(47.8 \%)$ of the patients receiving IVC and 7 (43.8\%) of the patients receiving MMF after 6 months of treatment $(p=0.961)$ and by $11(47.8 \%)$ of those who received IVC and 9 $(56.2 \%)$ of those who received MMF at 12 months of treatment $(p=0.713)$. Neither the PR rate nor the NR rate differed significantly at 6 or 12 months between the two groups. Conclusion. The efficacy of MMF does not differ from that of IVC in terms of induction of LN remission in Korean patients. (J Rheum Dis 2019;26:57-65)
\end{abstract}

Key Words. Lupus nephritis, Cyclophosphamide, Mycophenolic acid, Induction of remission

\section{INTRODUCTION}

Systemic lupus erythematosus (SLE) is a chronic, multi-organ autoimmune disease characterized by the presence of autoantibodies and various clinical manifestations. Renal injury is one of the most common and serious manifestations of SLE. Lupus nephritis (LN) develops in up to $60 \%$ of patients during the course of SLE, and it is the principal cause of mortality and morbidity because of the associated complications and progression to end-stage renal disease [1].

Many immunosuppressive agents have been developed to inhibit the progression of $\mathrm{LN}$ patients to renal failure. Conventionally, active proliferative $\mathrm{LN}$ has been managed using high doses of intravenous cyclophosphamide (IVC) combined with corticosteroids [2]. IVC has improved LN outcomes, but its use is limited by its severely toxic side effects, which can lead to hemorrhagic cystitis, opportunistic infections, bone marrow suppression, premature gonadal failure, and increased risk for malignancy [3]. To avoid such serious side effects, alternative treatments have been sought. Of the various immunosuppressants, mycophenolate mofetil (MMF) has emerged as a useful therapeutic modality. A randomized, controlled trial per-

Received : August 13, 2018, Revised : (1st) October 18, 2018, (2nd) November 12, 2018, Accepted : November 13,2018

Corresponding to : Shin-Seok Lee (iD http://orcid.org/0000-0001-6810-7355

Division of Rheumatology, Department of Internal Medicine, Chonnam National University Medical School \& Hospital, 42 Jebong-ro, Dong-gu, Gwangju 61469, Korea. E-mail : shinseok@chonnam.ac.kr

*These authors contributed equally to this work.

Copyright (c) 2019 by The Korean College of Rheumatology. All rights reserved.

This is a Open Access article, which permits unrestricted non-commerical use, distribution, and reproduction in any medium, provided the original work is properly cited. 
formed in 2005 showed that MMF was superior to IVC in terms of inducing a complete response (CR) in patients with active $\mathrm{LN}$, and it had a more favorable safety profile [4]. However, other studies and meta-analyses have not found significant differences between MMF and IVC in these contexts; both treatments have been associated with clinical improvements [5,6]. Notably, the Aspreva Lupus Management Study (ALMS) tested the hypothesis that MMF was superior to IVC as an LN remission induction therapy but found that the two treatments did not differ in terms of renal response or safety profile [7]. Interestingly, the ALMS found that the efficacy of IVC varied among different racial and ethnic groups, being less effective for patients of African and Hispanic descent [8]. MMF and IVC demonstrated similar efficacy and were used as induction treatment in previous trials in Hong Kong [9,10], Malaysia [11], China [12,13], Japan [14], and India $[15,16]$. Unfortunately, no randomized controlled trials have compared MMF and IVC to induce remission in Korean patients. Thus, in the present study, we retrospectively compared the efficacy of MMF and IVC as LN remission induction treatments in ethnically homogeneous Korean patients.

\section{MATERIALS AND METHODS}

\section{Patients}

This study was a retrospective observational study. Between January 2008 and January 2012, we evaluated 79 patients with LN from the lupus cohort of Chonnam National University Hospital. All patients fulfilled the revised 1997 criteria for SLE [17]. The inclusion criteria included a visit to our hospital within 6 months of SLE diagnosis, presence of adequate renal biopsy material, and a renal biopsy report suggestive of LN. Renal biopsy reports were used to confirm LN in all patients, and renal biopsy specimens were reclassified using the system of the International Society of Pathology/Renal Pathology Society (ISN/RPS) [18] by two renal pathologists blinded to previous biopsy data and clinical features. Patients were excluded if they exhibited advanced comorbidities or other diseases associated with kidney dysfunction, including diabetic or primary kidney disease. Patients for whom medical records were inadequate or who were followed up for $<1$ year were also excluded. Finally, of the 79 patients, we selected 39 patients with class III and IV lupus nephritis who received MMF or IVC as an LN induction therapy. The study was approved by the
Institutional Review Board of Chonnam National University Hospital (approval no. CNUN-2014-239), which waived the need for informed consent because of the retrospective nature of the study.

\section{Data collection}

Baseline demographic and clinical data were collected from medical records created at the time of renal biopsy. Demographic data (age, sex, educational level, hypertension and diabetes mellitus status, and disease duration at LN onset) were included. Hypertension was defined as systolic blood pressure $>140 \mathrm{mmHg}$ and/or diastolic blood pressure $>90 \mathrm{mmHg}$ on two or more occasions and/or the use of anti-hypertensive drugs. Diabetes mellitus was considered present if the fasting blood glucose level was $>126 \mathrm{mg} / \mathrm{dL}$ or the patient receiving insulin or taking oral hypoglycemic agent.

Laboratory data were based on a complete differential blood count, the erythrocyte sedimentation rate (ESR), C-reactive protein (CRP), urinalysis data, proteinuria (g/day) data, lipid profiles (levels of total cholesterol, high-density lipoprotein [HDL] cholesterol, low-density lipoprotein [LDL] cholesterol, and triglycerides), and the serum levels of albumin, and creatinine. Kidney function parameters were measured every $1 \sim 3$ months during follow-up. The glomerular filtration rate (GFR) was calculated according to the Modification of Diet in Renal Disease (MDRD) study equation: estimated GFR (eGFR, $\left.\mathrm{mL} / \mathrm{min} / 1.73 \mathrm{~m}^{2}\right)=186 \times\left(\mathrm{S}_{\mathrm{Cr}}[\mathrm{mg} / \mathrm{dL}]\right)^{-1154} \times(\text { age })^{-0203} \times$ (0.742 if female). Serological markers (the levels of autoantibodies, complement components [C3, C4, and CH50], anti-phospholipid antibodies [lupus anticoagulant, IgG/M anticardiolipin (aCL), and IgG anti-beta2-glycoprotein I $\left.\left(\beta_{2} \mathrm{GPI}\right)\right]$ ) were also measured. The levels of autoantibodies, including anti-Smith $(\mathrm{Sm})$, anti-ribonucleoprotein (RNP), anti-Ro, and anti-La autoantibodies, were assessed using enzyme-linked immunosorbent assays (ELISAs). Anti-nucleosome and anti-ribosomal P antibodies were measured using anti-extractable nuclear antigen (ENA). Disease activity was assessed using the SLE disease activity index (SLEDAI) 2000 [19].

The renal biopsy results were separately classified by two blinded renal pathologists based on the 2004 ISN/RPS criteria [18]. Activity and chronicity indices were determined by reference to the scoring systems of the US National Institutes of Health [20]. Patients with mixed-type $\mathrm{LN}$ were assigned to the predominant type. For example, type III+V cases were classified as type III 
and type IV+V cases as type IV.

We recorded medications used as induction and maintenance therapies, as well as all medications taken for $>3$ months prior to $\mathrm{LN}$ onset, such as hydroxychloroquine (HCQ) and prednisolone (>5 mg/day). We reviewed whether HCQ was taken continuously (for $>8$ months of the 1-year follow-up period) after LN onset. The use of angiotensin converting enzyme inhibitors (ACEis) and/or angiotensin receptor blockers (ARBs) (which reduce proteinuria) was also reviewed.

\section{Treatment and definition of remission}

Treatment was at the discretion of the responsible rheumatologist and included corticosteroids with high-dose

Table 1. Demographic and laboratory characteristics of the two different treatment groups at the time of renal biopsy

\begin{tabular}{|c|c|c|c|}
\hline Variables & IVC group $(\mathrm{n}=23)$ & MMF group $(n=16)$ & p-value \\
\hline Age at onset of LN (yr) & $31.7 \pm 8.86$ & $32.3 \pm 14.33$ & 0.832 \\
\hline Female, sex & 20/23 (87.0) & $15 / 16(93.8)$ & 0.452 \\
\hline Disease duration at onset of LN (mo) & $4.87 \pm 4.25$ & $7.44 \pm 5.50$ & 0.143 \\
\hline Education $(y r)$ & $12.78 \pm 3.75$ & $13.25 \pm 3.24$ & 0.789 \\
\hline Hypertension at onset of LN & $3 / 23(13.0)$ & $4 / 16(25.0)$ & 0.294 \\
\hline Diabetes mellitus at onset of $L N$ & $1 / 23(4.0)$ & $0 / 16(0)$ & 0.590 \\
\hline SLEDAI-2000 & $12.13 \pm 4.79$ & $11.12 \pm 4.25$ & 0.437 \\
\hline \multicolumn{4}{|l|}{ Laboratory findings } \\
\hline White blood cells $\left(/ \mathrm{mm}^{3}\right)$ & $5,634.8 \pm 2,913.7$ & $8,468.8 \pm 4,962.9$ & 0.050 \\
\hline Lymphocytes $\left(/ \mathrm{mm}^{3}\right)$ & $1,143.0 \pm 523.1$ & $1,474.4 \pm 992.6$ & 0.251 \\
\hline Hemoglobin $(g / d L)$ & $10.2 \pm 2.00$ & $11.3 \pm 1.46$ & 0.074 \\
\hline Platelets $\left(\times 10^{3} / \mathrm{mm}^{3}\right)$ & $196.3 \pm 74.5$ & $214.3 \pm 74.5$ & 0.471 \\
\hline $\mathrm{ESR}(\mathrm{mm} / \mathrm{h})$ & $38.5 \pm 28.8$ & $46.3 \pm 40.1$ & 0.662 \\
\hline $\mathrm{CRP}(\mathrm{mg} / \mathrm{dL})$ & $0.53 \pm 0.44$ & $0.75 \pm 0.84$ & 0.976 \\
\hline Albumin (mg/dL) & $2.86 \pm 0.77$ & $3.26 \pm 0.74$ & 0.168 \\
\hline Total cholesterol (mg/dL) & $198.8 \pm 48.1$ & $212.9 \pm 59.5$ & 0.582 \\
\hline HDL cholesterol (mg/dL) & $48.9 \pm 17.3$ & $57.8 \pm 20.7$ & 0.128 \\
\hline LDL cholesterol (mg/dL) & $123.1 \pm 43.4$ & $133.1 \pm 49.8$ & 0.329 \\
\hline Triglycerides (mg/dL) & $159.4 \pm 57.2$ & $154.6 \pm 56.6$ & 0.899 \\
\hline Serum creatinine $(\mathrm{mg} / \mathrm{dL})$ & $0.92 \pm 0.80$ & $0.85 \pm 0.39$ & 0.810 \\
\hline eGFR $\left(\mathrm{mL} / \mathrm{min} / 1.72 \mathrm{~m}^{2}\right)$ & $104.7 \pm 37.8$ & $103.4 \pm 43.8$ & 0.582 \\
\hline Proteinuria (g/24 h) & $3.34 \pm 3.12$ & $4.24 \pm 3.34$ & 0.315 \\
\hline \multicolumn{4}{|l|}{ Autoantibodies } \\
\hline Antinuclear & $23 / 23(100)$ & $15 / 16(93.8)$ & 0.410 \\
\hline Anti-dsDNA & $521.9 \pm 1,303.7$ & $267.0 \pm 763.7$ & 0.065 \\
\hline Anti-Sm & 9/23 (39.1) & 7/16 (43.8) & 0.515 \\
\hline Anti-RNP & $8 / 23(34.8)$ & $8 / 16(50.0)$ & 0.267 \\
\hline Anti-Ro/SS-A & $14 / 23(60.9)$ & $11 / 16(68.8)$ & 0.437 \\
\hline Anti-La/SS-B & $6 / 23(26.1)$ & 3/16 (18.8) & 0.446 \\
\hline Anti-nucleosome & $14 / 20(70.0)$ & $10 / 15(66.7)$ & 0.560 \\
\hline Anti-ribosomal-P & $5 / 20(25.0)$ & 4/15 (26.7) & 0.606 \\
\hline Lupus anticoagulant & $0 / 21(0.0)$ & $1 / 15(6.7)$ & 0.417 \\
\hline IgG-aCL & $2 / 21(9.5)$ & 3/15 (20.0) & 0.337 \\
\hline IgM-aCL & $0 / 20(0.0)$ & $0 / 14(0)$ & 1.000 \\
\hline $\operatorname{lgG}$ anti- $\beta_{2} \mathrm{GP} 1$ & 6/19 (31.6) & 8/14 (57.1) & 0.133 \\
\hline \multicolumn{4}{|l|}{ Complement levels } \\
\hline $\mathrm{C} 3$ & $47.2 \pm 24.0$ & $67.9 \pm 22.8$ & 0.011 \\
\hline $\mathrm{C} 4$ & $9.6 \pm 5.0$ & $19.3 \pm 24.9$ & 0.207 \\
\hline $\mathrm{CH} 50$ & $21.4 \pm 15.4$ & $29.2 \pm 14.9$ & 0.107 \\
\hline
\end{tabular}

Values are presented as mean \pm standard deviation or number (\%). IVC: intravenous cyclophosphamide, MMF: mycophenolate mofetil, LN: lupus nephritis, SLEDAI: systemic lupus erythematosus disease activity index, ESR: erythrocyte sedimentation rate, CRP: C-reactive protein, HDL: high-density lipoprotein, LDL: low-density lipoprotein, eGFR: estimated glomerular filtration rate, aCL: anti-cardiolipin, $\beta_{2} \mathrm{GPI}$ : beta2-glycoprotein I. 
IVC (500 1,000 mg/m $\mathrm{m}^{2}$ body surface area in monthly pulses) or corticosteroids with MMF (up to $3 \mathrm{~g} /$ day), followed by quarterly IVC or MMF [21,22]. Corticosteroids were administered as prednisolone $30 \sim 60 \mathrm{mg} /$ day, with or without intravenous methylprednisolone pulse therapy (500 1,000 mg/day $\times 3$ days).

Treatment responses after 6 and 12 months were defined using the ACR 2006 clinical trial criteria [23]. A CR was defined as a normal GFR $\left(90 \mathrm{~mL} / \mathrm{min} / 1.73 \mathrm{~m}^{2}\right)$ or a $>25 \%$ increase from baseline or, if the baseline estimated GFR (eGFR) was abnormal, as a urine protein-to-creatinine ratio $<0.2$, a dipstick test result of 0 or trace, and no urinary sediment ( $\leq 5$ red blood cells [RBCs]/ high-power field [HPF] and no cellular casts). A partial response (PR) was defined as a stable eGFR, a $>50 \%$ reduction in the urinary protein-to-creatinine ratio (or a ratio in the range of $0.2 \sim 2.0$ ), and no urinary sediment. No response (NR) was defined as a failure to meet the remission criteria.

\section{Statistical analysis}

All statistical analyses and data processing were performed using SPSS software (ver. 23.0; IBM Co., Armonk, NY, USA). Continuous variables are expressed as mean \pm standard deviation, and categorical variables as percentages. The Mann-Whitney U-test was used to compare continuous variables, and chi-square test and Fisher's exact tests were used to compare categorical variables. The Mantel-Haenszel method was employed to compare the renal responses of the IVC and MMF groups at 6 and 12 months. p-values $<0.05$ were considered to be statistically significant.

\section{RESULTS}

We included a total of 39 patients with biopsy-proven $\mathrm{LN}$. The mean age at $\mathrm{LN}$ onset was $31.92 \pm 11.2$ years, and $89.7 \%$ of all the patients were women. The mean disease duration at the time of biopsy-confirmed LN onset was $5.92 \pm 4.90$ months. Among the patients, $17.9 \%$ had hypertension at the time of $\mathrm{LN}$ onset, and $2.6 \%$ diabetes mellitus. In all, 23 patients (59.0\%) were treated with IVC as induction therapy, and $16(41.0 \%)$ were treated with MMF; both groups were also prescribed corticosteroids.

The baseline demographic and clinical characteristics of all the patients at the time of renal biopsy are shown in Table 1. The mean age at $\mathrm{LN}$ onset was 31.7 years in the IVC-treated patients and 32.3 years in the MMF-treated patients. The mean disease duration at the time of $\mathrm{LN}$ onset was 4.87 months in the IVC-treated patients and 7.44 months in the MMF-treated patients. In terms of laboratory findings, the IVC-treated patients had lower white blood cell counts $(p=0.050)$ than the MMF-treated patients. No other laboratory measurements (of inflammatory markers, lipid profiles, or kidney function pa-

Table 2. Renal biopsy findings in the two different treatment groups

\begin{tabular}{|c|c|c|c|}
\hline Variable & IVC group $(n=23)$ & MMF group $(n=16)$ & p-value \\
\hline \multicolumn{4}{|l|}{ Activity index } \\
\hline Endo-capillary hypercellularity & $22(95.7)$ & $12(75.0)$ & 0.080 \\
\hline Leukocyte infiltration & $19(82.6)$ & $12(75.0)$ & 0.425 \\
\hline Sub-endothelial hyaline deposits & $22(95.7)$ & $14(87.5)$ & 0.363 \\
\hline Fibrinoid necrosis/Karyorrhexis & $19(82.6)$ & $7(43.8)$ & 0.014 \\
\hline Cellular crescents & $9(39.1)$ & $4(25.0)$ & 0.285 \\
\hline Interstitial inflammation & $22(95.7)$ & $15(93.8)$ & 0.659 \\
\hline \multicolumn{4}{|l|}{ Chronicity index } \\
\hline Glomerular sclerosis & $7(30.4)$ & $9(56.2)$ & 0.100 \\
\hline Tubular atrophy & $13(56.5)$ & $12(75.0)$ & 0.200 \\
\hline Interstitial fibrosis & $17(73.9)$ & $13(81.2)$ & 0.446 \\
\hline Fibrous crescents & $2(8.7)$ & $2(12.5)$ & 0.548 \\
\hline Activity score & $9.65 \pm 3.10$ & $6.25 \pm 3.72$ & 0.007 \\
\hline Active score $(\geq 12)$ & $9(39.1)$ & $2(12.5)$ & 0.070 \\
\hline Chronicity score & $1.87 \pm 1.33$ & $2.50 \pm 1.55$ & 0.159 \\
\hline Chronic score $(\leq 4)$ & $3(13.0)$ & $6(37.5)$ & 0.082 \\
\hline
\end{tabular}

Values are presented as number (\%) or mean \pm standard deviation. IVC: intravenous cyclophosphamide, MMF: mycophenolate mofetil. 
rameters) differed between the two groups. The autoantibody levels did not differ between the two groups. In terms of complement components, the $\mathrm{C} 3$ level was significantly lower in the IVC-treated patients than in the MMF-treated patients $(\mathrm{p}=0.011)$.

The renal biopsy findings of the two groups are compared in Table 2. Of the various activity indices, fibrinoid necrosis/karyorrhexis was more common in the IVC-treated patients than in the MMF-treated patients $(p=0.014)$. Similarly, the activity score was significantly higher in the IVC-treated patients than in the MMF-treated patients $(p=0.007)$. No other chronicity indices or scores differed between the two groups. However, when patients with an activity score greater than 12 were evaluated, there was no difference in number of patients between the two treatment groups. To conclude, the renal responses for the two different treatments at 6 and 12 months in patients with higher activity scores in the renal biopsy (activity score $>12$ ) were not significantly different ( $p=0.973$ for the 6 -month outcome, $p=0.708$ for the 12-month outcome).

Table 3 lists the medications taken before $\mathrm{LN}$ onset and during the follow-up period. There were no significant between-group differences in the use of prednisolone, HCQ, ACEi, or ARB. In addition, the medications used during follow-up, including $\mathrm{HCQ}, \mathrm{ACEi}$, and ARB, did not differ between the groups. During induction treatment, two patients from IVC group were treated with steroid pulse therapy. However, no patient in MMF group received steroid pulse therapy. For maintenance therapy, IVC, MMF, Azathioprine (AZA), and glucocorticoids were considered according to the ACR guidelines for the management of lupus nephritis class III/IV induction therapy. In this study, as maintenance therapy, 16/16 (100\%) patients in the MMF group treated with MMF as maintenance therapy. In the IVC group, 4/23 (17.4\%) pa-

Table 3. Medications used by LN patients in the two different treatment groups

\begin{tabular}{lccc}
\hline \multicolumn{1}{c}{ Variable } & IVC group $(\mathrm{n}=23)$ & MMF group $(\mathrm{n}=16)$ & $\mathrm{p}$-value \\
\hline Treatment before diagnosis of $\mathrm{NN}$ & & & \\
Prednisolone $(>5 \mathrm{mg} /$ day) & $12(52.1)$ & $12(75.0)$ & 0.134 \\
Hydroxychloroquine & $12(52.1)$ & $10(62.5)$ & 0.379 \\
ACEi or ARB & $2(8.7)$ & $0(0.0)$ & 0.341 \\
Hydroxychloroquine during follow-up & $19(82.6)$ & $15(93.8)$ & 0.305 \\
ACEi or ARB during follow-up & $16(69.6)$ & $8(50.0)$ & 0.184 \\
\hline
\end{tabular}

Values are presented as number (\%). LN: lupus nephritis, IVC: intravenous cyclophosphamide, MMF: mycophenolate mofetil, ACEi: angiotensin converting enzyme inhibitor, ARB: angiotensin receptor blocker.

Table 4. Renal responses in the two different treatment groups at 6 and 12 months

\begin{tabular}{|c|c|c|c|}
\hline Variable & IVC group $(n=23)$ & MMF group $(n=16)$ & $\mathrm{p}$-value \\
\hline 6-month outcome & & & 0.961 \\
\hline $\mathrm{CR}$ & $11(47.8)$ & $7(43.8)$ & \\
\hline PR & $5(21.7)$ & $4(25.0)$ & \\
\hline NR & $7(30.4)$ & $5(31.2)$ & \\
\hline 12-month outcome & & & 0.713 \\
\hline $\mathrm{CR}$ & $11(47.8)$ & $9(56.2)$ & \\
\hline PR & $7(30.4)$ & $3(18.8)$ & \\
\hline NR & $5(21.7)$ & $4(25.0)$ & \\
\hline Reduction of $24 \mathrm{~h}$ proteinuria, $>50 \%$ & $18(78.3)$ & $12(75.0)$ & 0.554 \\
\hline Time to reduction, $>50 \%$, days & $145.3 \pm 170.8$ & $198.9 \pm 231.7$ & 0.917 \\
\hline Reduction of proteinuria, $<500 \mathrm{mg} /$ day & $16(69.6)$ & $12(75.0)$ & 0.500 \\
\hline Time to reduction, $<500 \mathrm{mg} /$ day, days & $257.7 \pm 341.8$ & $318.3 \pm 288.7$ & 0.537 \\
\hline Reduction of proteinuria, $<200 \mathrm{mg} /$ day & $14(60.9)$ & $10(62.5)$ & 0.593 \\
\hline Time to reduction, $<200 \mathrm{mg} /$ day, days & $486.0 \pm 515.6$ & $609.2 \pm 433.7$ & 0.335 \\
\hline
\end{tabular}

Values are presented as number (\%) or mean \pm standard deviation. IVC: intravenous cyclophosphamide, MMF: mycophenolate mofetil, CR: complete response, PR: partial response, NR: no response. 
tients received quarterly IVC, 5/23 (21.7\%) received MMF, 7/23 (30.4\%) received AZA, and 5/23 (21.7\%) received steroids as maintenance medication.

The renal responses of the two groups at 6 and 12 months are presented in Table 4. At 6 months, 11 of the 23 IVC-treated patients $(47.8 \%)$ and 7 of the 16 MMF-treated patients (43.8\%) had attained a CR. PRs were evident in 5 of the 23 IVC-treated patients (21.7\%) and 4 of the 16 MMF-treated patients (25.0\%). The renal responses at 6 months did not differ significantly between the groups ( $\mathrm{p}=0.961)$. At 12 months, $11(47.8 \%)$ and 7 (30.4\%) of the IVC-treated patients exhibited either a CR or a PR, respectively, as did 9 (56.2\%) and 3 (18.8\%) of the MMF-treated patients. Similarly, the renal responses at 12 months did not differ significantly between the two groups $(p=0.713)$. The renal outcomes at 6 and 12 months were defined using the ACR 2006 response criteria. Renal dysfunction (GFR $<60$ ) at the secondary endpoint occurred in 3/23 (13.0\%) IVC patients and 5/16 (31.3\%) MMF patients. No other chronicity indices or scores differed between the two groups.

Additional analyses were performed of patients who exhibited a renal response, defined as a $>50 \%$ reduction of 24 hours proteinuria, $<500 \mathrm{mg} /$ day proteinuria, or $<200 \mathrm{mg}$ /day proteinuria (Table 4). Importantly, similar numbers of patients in the two groups met these criteria; no significant between-group differences were apparent. Furthermore, the time until proteinuria reduction, defined as a $>50 \%$ reduction of 24 hours proteinuria, $<500$ $\mathrm{mg} /$ day proteinuria, or $<200 \mathrm{mg} /$ day proteinuria, did not differ between the groups.

The adverse events experienced by the patients over 12 months are shown in Table 5. No patients died during the treatment period. The incidence of adverse events was similar in the two groups: $65.2 \%$ (15 of the 23 IVC-treated patients) and $68.8 \%$ (11 of the 16 MMF-treated patients, $\mathrm{p}=0.548$ ). The proportion of patients with major infections requiring hospitalization did not differ significantly between the groups $(p=0.061)$.

\section{DISCUSSION}

We found no significant differences between IVC and MMF used as remission induction therapies for LN. In other words, MMF and IVC were equally effective in terms of inducing remission in an ethnically homogenous Korean population.

According to the $\mathrm{LN}$ management guidelines proposed by the ACR, European League Against Rheumatism (EULAR), and Kidney Disease Improving Global Outcomes (KDIGO) $[21,22,24]$, patients with active LN are recommended to take IVC or MMF in combination with oral glucocorticoids, with or without three pulses of intravenous methylprednisolone at the start of remission induction therapy. Because these guidelines are based on clinical trials conducted in Western countries and treatment responses vary by geographical region, race, and ethnicity, it is important to perform randomized, controlled trials in Asian populations to derive meaningful guidelines. Unfortunately, a few studies have yet compared the efficacy and safety of IVC and MMF as remission induction therapies in Asian. Although our work was retrospective in nature, this report is the first to compare the efficacy of IVC and MMF in Korean patients with $\mathrm{LN}$. We believe that our results could guide the management of LN patients in our region. However, because of

Table 5. Adverse events in the two different treatment groups over 12 months

\begin{tabular}{|c|c|c|c|}
\hline Variable & IVC group $(n=23)$ & MMF group $(n=16)$ & $\mathrm{p}$-value \\
\hline Any adverse event & $15(65.2)$ & $11(68.8)$ & 0.548 \\
\hline Death & $0(0.0)$ & $0(0.0)$ & - \\
\hline \multicolumn{4}{|l|}{ Major infection requiring hospitalization } \\
\hline Pneumonia & $0(0.0)$ & $3(18.8)$ & 0.061 \\
\hline \multicolumn{4}{|l|}{ Minor infection } \\
\hline Cellulitis & $1(4.3)$ & $0(0.0)$ & 0.590 \\
\hline Herpes zoster & $1(4.3)$ & $1(6.2)$ & 0.659 \\
\hline Upper respiratory tract infection & $2(8.6)$ & $2(12.5)$ & 0.548 \\
\hline Amenorrhea & $0(0.0)$ & $1(6.2)$ & 0.410 \\
\hline Menstrual irregularities & $2(8.6)$ & $0(0.0)$ & 0.341 \\
\hline Leukopenia, WBC count $<1,500 / \mu \mathrm{L}$ & $2(8.6)$ & $0(0.0)$ & 0.341 \\
\hline
\end{tabular}

Values are presented as number (\%). IVC: intravenous cyclophosphamide, MMF: mycophenolate mofetil, WBC: white blood cell. 
the small sample size, a larger-scale, multi-center, nationwide prospective study is needed to confirm our findings. We found that MMF and IVC were similarly effective when used as remission induction therapy for management of active LN. In 2012, Li et al. [13] conducted 24-week prospective study in China, including 60 patients with LN randomly assigned to receive MMF, tacrolimus, or IVC in combination with corticosteroids. This pilot study suggested that both MMF and tacrolimus are viable alternatives to IVC as induction therapies for acute LN in Chinese patients. In 2018, Sahay et al. [16] compared the efficacy and side effects of cyclophosphamidebased (low- and high- dose) and MMF-based regimens with $144 \mathrm{LN}$ patients in India. The MMF- and IVC-based regimens were equally effective for the treatment of $\mathrm{LN}$. Moreover, this study suggested that the low-dose IVC regime may be equally efficacious, but with a further reduction in cost and drug toxicity. In 2005, a 24-week, randomized, open-label, non-inferiority trial showed that remission induction therapy with MMF was superior to that afforded by IVC in inducing a CR [4]. In contrast, the multinational ALMS study found that renal responses to MMF remission induction therapy were comparable to those afforded by IVC at 6 months [7]. Although a tendency toward a better response with IVC rather than with MMF was evident when the analyses were limited to Asian patients, statistical significance was not attained. Hanaoka et al. [14] recently conducted a single-center retrospective study comparing four different induction therapies in Japanese LN patients, and they found that the $\mathrm{CR}$ rate over 3 years did not differ significantly among the groups. When the renal responses of 22 IVC-treated patients were compared to those of 11 MMF-treated patients, no significant between-group differences were apparent.

Our results are in agreement with those of the ALMS and Japanese studies. Because our work was retrospective in nature, some selection bias might have occurred. Although the baseline clinical characteristics, except complement level, did not differ between the two groups, the activity score derived from renal pathology was significantly higher in the IVC group. Thus, we adjusted for the activity score, but the renal responses at 6 and 12 months did not change. A randomized, controlled trial is better than a retrospective study. However, it is difficult to conduct clinical trials with SLE patients to issues including disease heterogeneity, inadequate trial size, short trial duration, insufficient information on appropriate dosage or guidelines to address background medications, and difficulty in setting a primary endpoint [25]. Thus, we must use information derived from observational studies. We found that MMF could be a good therapeutic option for $\mathrm{LN}$ patients, particularly women of childbearing age, because it does not cause gonadal toxicity. Ideally, decision-making should be shared by physicians and patients, who together choose an appropriate regimen by balancing the benefits and risks of treatment.

In this study, we did not detect significant differences between the MMF- and IVC-treated patients with regard to the rates of adverse events, serious adverse events, or infections. Although our study was retrospectively analyzed, the overall adverse event profiles of both MMF and IVC in this study were consistent with previous studies [4,7,26-28]. However, it should be noted that under-reporting of some adverse events might have occurred due to the retrospective study design.

Our study had several limitations. First, it was a retrospective study, which might limit the generalizability of the results. Second, the number of $\mathrm{LN}$ patients allocated to each treatment arm was relatively small, creating power issues in terms of analysis. Any difference in the renal response between the IVC- and MMF-treated patients may have failed to attain statistical significance because of the small sample size. Third, the IVC-treated patients had more active disease compared to the MMF-treated patients; the $\mathrm{C} 3$ level, fibrinoid necrosis/karyorrhexis components of the activity indices, and activity scores were significantly higher in the IVC-treated patients than in the MMF-treated patients. Although we adjusted for the activity scores, some potential confounding factors could not be fully controlled because of the nature of the study. Finally, the work was conducted at a single center in the Republic of Korea, and thus, the results may not be representative of the entire SLE population of Asian ethnicity. Therefore, a larger-scale, multi-center, nationwide prospective study is needed to confirm our findings.

\section{CONCLUSION}

In conclusion, we found that the efficacy and safety of MMF at 6 and 12 months did not differ from that of IVC when the drugs were used as LN induction treatments in ethnically homogeneous Korean patients. Both IVC and MMF can be used for LN remission therapy. 


\section{ACKNOWLEDGMENTS}

This work was supported by the Bio \& Medical Technology Development Program of the NRF funded by the Korean government, MSIP (2017M3A9E8023014), and by a grant (CRI13904-24 and CRI18043-21) from the Chonnam National University Hospital Biomedical Research Institute.

\section{CONFLICT OF INTEREST}

No potential conflict of interest relevant to this article was reported.

\section{REFERENCES}

1. Tsokos GC. Systemic lupus erythematosus. N Engl J Med 2011;365:2110-21.

2. Illei GG, Austin HA, Crane M, Collins L, Gourley MF, Yarboro $\mathrm{CH}$, et al. Combination therapy with pulse cyclophosphamide plus pulse methylprednisolone improves long-term renal outcome without adding toxicity in patients with lupus nephritis. Ann Intern Med 2001;135:248-57.

3. Mok CC, Chan PT, To CH. Anti-müllerian hormone and ovarian reserve in systemic lupus erythematosus. Arthritis Rheum 2013;65:206-10.

4. Ginzler EM, Dooley MA, Aranow C, Kim MY, Buyon J, Merrill JT, et al. Mycophenolate mofetil or intravenous cyclophosphamide for lupus nephritis. N Engl J Med 2005;353:2219-28.

5. Kamanamool N, McEvoy M, Attia J, Ingsathit A, Ngamjanyaporn P, Thakkinstian A. Efficacy and adverse events of mycophenolate mofetil versus cyclophosphamide for induction therapy of lupus nephritis: systematic review and meta-analysis. Medicine (Baltimore) 2010;89:227-35.

6. Touma Z, Gladman DD, Urowitz MB, Beyene J, Uleryk EM, Shah PS. Mycophenolate mofetil for induction treatment of lupus nephritis: a systematic review and metaanalysis. J Rheumatol 2011;38:69-78.

7. Appel GB, Contreras G, Dooley MA, Ginzler EM, Isenberg D, Jayne D, et al. Mycophenolate mofetil versus cyclophosphamide for induction treatment of lupus nephritis. J Am Soc Nephrol 2009;20:1103-12.

8. Isenberg D, Appel GB, Contreras G, Dooley MA, Ginzler EM, Jayne D, et al. Influence of race/ethnicity on response to lupus nephritis treatment: the ALMS study. Rheumatology (Oxford) 2010;49:128-40.

9. Chan TM, Li FK, Tang CS, Wong RW, Fang GX, Ji YL, et al. Efficacy of mycophenolate mofetil in patients with diffuse proliferative lupus nephritis. Hong Kong-Guangzhou nephrology study group. N Engl J Med 2000;343:1156-62.

10. Chan TM, Tse KC, Tang CS, Mok MY, Li FK; Group HKNS. Long-term study of mycophenolate mofetil as continuous induction and maintenance treatment for diffuse proliferative lupus nephritis. J Am Soc Nephrol 2005;16: 1076-84.

11. Ong LM, Hooi LS, Lim TO, Goh BL, Ahmad G, Ghazalli R, et al. Randomized controlled trial of pulse intravenous cyclophosphamide versus mycophenolate mofetil in the induction therapy of proliferative lupus nephritis. Nephrology (Carlton) 2005;10:504-10.

12. Hu W, Liu Z, Chen H, Tang Z, Wang Q, Shen K, et al. Mycophenolate mofetil vs cyclophosphamide therapy for patients with diffuse proliferative lupus nephritis. Chin Med J (Engl) 2002;115:705-9.

13. Li X, Ren H, Zhang Q, Zhang W, Wu X, Xu Y, et al. Mycophenolate mofetil or tacrolimus compared with intravenous cyclophosphamide in the induction treatment for active lupus nephritis. Nephrol Dial Transplant 2012;27: 1467-72.

14. Hanaoka H, Kiyokawa T, Iida H, Ishimori K, Takakuwa Y, Okazaki T, et al. Comparison of renal response to four different induction therapies in Japanese patients with lupus nephritis class III or IV: a single-centre retrospective study. PLoS One 2017;12:e0175152.

15. Rathi M, Goyal A, Jaryal A, Sharma A, Gupta PK, Ramachandran $\mathrm{R}$, et al. Comparison of low-dose intravenous cyclophosphamide with oral mycophenolate mofetil in the treatment of lupus nephritis. Kidney Int 2016;89: 235-42.

16. Sahay M, Saivani Y, Ismal K, Vali PS. Mycophenolate versus cyclophosphamide for lupus nephritis. Indian J Nephrol 2018;28:35-40.

17. Hochberg MC. Updating the American College of Rheumatology revised criteria for the classification of systemic lupus erythematosus. Arthritis Rheum 1997;40:1725.

18. Weening JJ, D'Agati VD, Schwartz MM, Seshan SV, Alpers CE, Appel GB, et al. The classification of glomerulonephritis in systemic lupus erythematosus revisited. Kidney Int 2004;65:521-30.

19. Gladman DD, Ibañez D, Urowitz MB. Systemic lupus erythematosus disease activity index 2000. J Rheumatol 2002;29: 288-91.

20. Austin HA 3rd, Muenz LR, Joyce KM, Antonovych TA, Kullick ME, Klippel JH, et al. Prognostic factors in lupus nephritis. Contribution of renal histologic data. Am J Med 1983;75:382-91.

21. Bertsias GK, Tektonidou M, Amoura Z, Aringer M, Bajema $\mathrm{I}$, Berden $\mathrm{JH}$, et al. Joint European League Against Rheumatism and European Renal Association-European Dialysis and Transplant Association (EULAR/ERA-EDTA) recommendations for the management of adult and paediatric lupus nephritis. Ann Rheum Dis 2012;71:1771-82.

22. Hahn BH, McMahon MA, Wilkinson A, Wallace WD, Daikh DI, Fitzgerald JD, et al. American College of Rheumatology guidelines for screening, treatment, and management of lupus nephritis. Arthritis Care Res (Hoboken) 2012;64: 797-808.

23. Renal Disease Subcommittee of the American College of Rheumatology Ad Hoc Committee on Systemic Lupus Erythematosus Response C. The American College of Rheumatology response criteria for proliferative and membranous renal disease in systemic lupus erythematosus clinical trials. Arthritis Rheum 2006;54:421-32.

24. Radhakrishnan J, Cattran DC. The KDIGO practice guideline on glomerulonephritis: reading between the (guide) lines--application to the individual patient. Kidney Int 2012;82:840-56. 
25. Mahieu MA, Strand V, Simon LS, Lipsky PE, RamseyGoldman R. A critical review of clinical trials in systemic lupus erythematosus. Lupus 2016;25:1122-40.

26. Moore RA, Derry S. Systematic review and meta-analysis of randomised trials and cohort studies of mycophenolate mofetil in lupus nephritis. Arthritis Res Ther 2006;8:R182.

27. Walsh M, James M, Jayne D, Tonelli M, Manns BJ,
Hemmelgarn BR. Mycophenolate mofetil for induction therapy of lupus nephritis: a systematic review and meta-analysis. Clin J Am Soc Nephrol 2007;2:968-75.

28. Zhu B, Chen N, Lin Y, Ren H, Zhang W, Wang W, et al. Mycophenolate mofetil in induction and maintenance therapy of severe lupus nephritis: a meta-analysis of randomized controlled trials. Nephrol Dial Transplant 2007;22:1933-42. 